\title{
Construction of Weighted Second Order Rotatable Simplex Designs (Wrsd)
}

\author{
Otieno-Roche Emily ${ }^{1}$, Koske Joseph ${ }^{2}$, Mutiso John ${ }^{2}$ \\ ${ }^{1}$ Department of Computer and Information Technology, Africa Nazarene University, Nairobi, Kenya \\ ${ }^{2}$ Department of Statistics and Computer Science, Moi University, Eldoret, Kenya
}

Email address:

rocheemmy@gmail.com (Otieno-Roche E.),koske4@yahoo.co.uk (Koske J.), johnkasome@yahoo.com (Mutiso J.)

To cite this article:

Otieno-Roche Emily, Koske Joseph, Mutiso John. Construction of Weighted Second Order Rotatable Simplex Designs (Wrsd). American Journal of Theoretical and Applied Statistics. Vol. 6, No. 6, 2017, pp. 303-310. doi: 10.11648/j.ajtas.20170606.17

Received: June 2, 2017; Accepted: June 16, 2017; Published: December 7, 2017

\begin{abstract}
Response surface methodology is widely used for developing, improving, and optimizing processes in various fields. A rotatable simplex design is one of the new designs that have been suggested for fitting second-order response surface models. In this article, we present a method for constructing weighted second order rotatable simplex designs (WRSD) which are more efficient than the ordinary rotatable simplex designs (RSD). Using moment matrices based on the Simplex and Factorial Designs, and the General Equivalence Theorem (GET) for D- and A- optimality, weighted rotatable simplex designs (WRSDs) were obtained. A- and D- optimality criterion was then used to establish the efficiency of the designs.
\end{abstract}

Keywords: D - Optimal, A - Optimal, Response Surface Designs, Second-Order Designs, Information Surface, Moment Matrices, Weighted Rotatable Simplex Designs

\section{Introduction}

A rotatable simplex design is one of the newly introduced designs in response surface experiment. Rotatable Simplex Designs have been suggested to have a very wide usage e.g in Food science, Business performance, Health sciences, Bioprocessing, Engineering, Construction Industry and so on as its performance was illustrated in Response Surface Analysis (RSA) of percentage crude oil removed by three factors. Rotatable Simplex Designs (RSDs) are constructed using the properties of a Simplex - lattice design (SLD), through Full Factorial Designs (FFDs)

Design points from SLD are used to generate the original design points for the RSD. The levels of the SLD were increased by taking all the combination levels of the original points from the SLD such that the sum of all odd moments is zero. These points were then augmented with all the combination levels of the distance from the centre point $(a)$. Equation (1) was then solved to attain rotatability.

$$
\sum t_{i u}^{4}=3 \sum t_{i u}^{2} t_{j u}^{2} \text { for all } i \neq j
$$

where the summation in the above relations is over the design points $u=1,2, \ldots, N$.
The introduction of weights to RSD in this paper is for the purpose of improving the design by making it more efficient

\section{Optimality Criteria and Efficiencies}

Optimal designs are experimental designs that are generated based on a particular optimality criterion and are generally optimal only for a specific statistical model. The ultimate purpose of any optimality criterion is to measure the largeness of a non-negative definite $s \times s$ information matrix C.

The optimality criterion used in this study were from the family of matrix means $\emptyset_{p}$ for $p=-1,0$, introduced by Kiefer (1964) and is discussed in detail by Pukelsheim (1993).

Efficiency tests the goodness of a design.

\section{Weighted Rotatable Simplex Designs}

A WRSD is modified from the general RSD by separating it into Simplex and "Radius" Factorial blocks having weights $\alpha_{1}$ and $\alpha_{2}$ assigned to the Simplex block $\left(\tau_{1}\right)$ and 'Radius' factorial block $\left(\tau_{2}\right)$.

In this study, the rotatable WRSD will be expressed as: 


$$
M(\mathrm{~T})=\alpha_{1} M\left(\tau_{1}\right)+\alpha_{2} M\left(\tau_{2}\right)
$$

An outline of the procedure to be used in obtaining the WRSDs is as follows:

\subsection{Construction of $\{2,2\}$ Wrsd}

The elementary RSD $\tau_{1}$ and $\tau_{2}$ are used to generate the WRSD $\mathrm{T}$ with points as given from [Table 1]

Table 1. Design points for $\{2,2\} R S D$.

\begin{tabular}{ccccccccccccc}
\hline$t_{1 u}$ & 1 & -1 & 0 & 0 & $1 / 2$ & $-1 / 2$ & $-1 / 2$ & $1 / 2$ & $a$ & $-a$ & $a$ & $-a$ \\
$t_{2 u}$ & 0 & 0 & 1 & -1 & $1 / 2$ & $-1 / 2$ & $1 / 2$ & $-1 / 2$ & $a$ & $a$ & $-a$ & $-a$ \\
\hline
\end{tabular}

$$
\text { Where } a=\left[\frac{3}{16}\right]^{\frac{1}{4}}
$$$$
\text { Thu } \tau_{1}=\left[\begin{array}{ccc}
t_{0 u} & t_{1 u} & t_{2 u} \\
1 & 1 & 0 \\
1 & 0 & 0 \\
1 & 0 & -1 \\
1 & \frac{1}{2} & \frac{1}{2} \\
1 & -\frac{1}{2} & -\frac{1}{2} \\
1 & -\frac{1}{2} & \frac{1}{2} \\
1 & \frac{1}{2} & -\frac{1}{2}
\end{array}\right] \text { and } \tau_{2}=\left[\begin{array}{ccc}
1 & a & a \\
1 & -a & a \\
1 & a & -a \\
1 & -a & -a
\end{array}\right]
$$

such that, for weights $\alpha_{1}, \alpha_{2} \geq 0$ with $\alpha_{1}+\alpha_{2}=1$, the design $\mathrm{T}=\alpha_{1} \tau_{1}+\alpha_{2} \tau_{2}$ has

$$
M\left(\tau_{1}\right)=\frac{1}{8}\left[\begin{array}{cccc}
8 & 31_{2}^{\prime} & 0 & 0 \\
31_{2} & \frac{9}{4} \mathrm{I}_{2}+\frac{1}{4}\left(e_{1} e_{2}^{\prime}+e_{2} e_{1}^{\prime}\right) & 0 & 0 \\
0 & 0 & 3 \mathrm{I}_{2} & 0 \\
0 & 0 & 0 & \frac{1}{4}
\end{array}\right]
$$

and

$$
M\left(\tau_{2}\right)=\frac{1}{4}\left[\begin{array}{cccc}
4 & \left(4 a^{2}\right) 1_{2}^{\prime} & 0 & 0 \\
\left(4 a^{2}\right) 1_{2} & \left(4 a^{4}\right) \mathrm{J}_{2} & 0 & 0 \\
0 & 0 & \left(4 a^{2}\right) \mathrm{I}_{2} & 0 \\
0 & 0 & 0 & 4 a^{4}
\end{array}\right]
$$

So that using (4) and (5) in (2) becomes

$$
M(\mathrm{~T})=\left[\begin{array}{cccc}
1 & b 1_{2}^{\prime} & 0 & 0 \\
b 1_{2} & c \mathrm{I}_{2}+d\left(e_{1} e_{2}^{\prime}+e_{2} e_{1}^{\prime}\right) & 0 & 0 \\
0 & 0 & b \mathrm{I}_{2} & 0 \\
0 & 0 & 0 & d
\end{array}\right]
$$

With its corresponding inverse as:

$$
(M(\mathrm{~T}))^{-1}=\left[\begin{array}{cccccc}
\frac{\left(c^{2}-d^{2}\right)}{\left|M_{1}\right|} & -\frac{b(c-d)}{\left|M_{1}\right|} & -\frac{b(c-d)}{\left|M_{1}\right|} & 0 & 0 & 0 \\
-\frac{b(c-d)}{\left|M_{1}\right|} & \frac{e c-b^{2}}{\left|M_{1}\right|} & \frac{b^{2}-e d}{\left|M_{1}\right|} & 0 & 0 & 0 \\
-\frac{b(c-d)}{\left|M_{1}\right|} & \frac{b^{2}-e d}{\left|M_{1}\right|} & \frac{e c-b^{2}}{\left|M_{1}\right|} & 0 & 0 & 0 \\
0 & 0 & 0 & \frac{1}{b} & 0 & 0 \\
0 & 0 & 0 & 0 & \frac{1}{b} & 0 \\
0 & 0 & 0 & 0 & 0 & \frac{1}{d}
\end{array}\right](7)
$$

where

$$
b=\frac{3 \alpha_{1}}{8}+a^{2} \alpha_{2}, \quad c=\frac{9 \alpha_{1}}{32}+a^{4} \alpha_{2}, \quad d=\frac{\alpha_{1}}{32}+a^{4} \alpha_{2} \text { and } \quad\left|M_{1}\right|=(c-d)\left[a(c+d)-2 b^{2}\right]
$$

\section{2. $\{2,2\}$ Optimal Designs}

Optimal designs are experimental designs that are generated based on a particular optimality criterion and are generally optimal only for a specific statistical model. Here we have used the general equivalence theorem to compute the values of the masses assigned to the design points of the RSD to obtain D-optimal and A-optimal WRSDs. Optimality measures the largeness of a non-negative definite $s \times s$ matrix $C$ where $C$ is the subset of $M$. In this study, the matrix $C$ will be the information matrix based on the full parameter system of the model.

\subsubsection{D-Optimal Wrsd}

$$
\operatorname{trace}\left(M\left(\tau_{i}\right)(M(\mathrm{~T}))^{-1}\right)=\operatorname{trace}(M(\mathrm{~T}))^{0}, \text { for } i=1,2
$$

Thus from (4) and (7), $M\left(\tau_{1}\right)(M(\mathrm{~T}))^{-1}$ becomes 


$$
\left[\begin{array}{cccc}
\frac{(c-d)(-4(c+d)+3 b)}{4\left|M_{1}\right|} & \left(\frac{(c-d)(-3+8 b)}{8\left|M_{1}\right|}\right) 1^{\prime}{ }_{2} & 0 & 0 \\
\left(\frac{(c-d)[-6(c+d)+5 b]}{16\left|M_{1}\right|}\right) 1_{2} & \left(\frac{-2 b^{2}+c+d}{4\left|M_{1}\right|}\right) \mathrm{I}_{2}+\left(-\frac{-8 b^{2}-c+12 b(c-d)+9 d}{32\left|M_{1}\right|}\right) \mathrm{J}_{2} & 0 & 0 \\
0 & 0 & \left(\frac{3}{8 b}\right) \mathrm{I}_{2} & 0 \\
0 & 0 & 0 & \frac{1}{32 d}
\end{array}\right]
$$

With its trace being

$$
\frac{1}{8}\left[8\left(\frac{\left(c^{2}-d^{2}\right)}{\left|M_{1}\right|}\right)+12\left(-\frac{b(c-d)}{\left|M_{1}\right|}\right)+\frac{9}{2}\left(\frac{e c-b^{2}}{\left|M_{1}\right|}\right)+\frac{1}{2}\left(\frac{b^{2}-e d}{\left|\boldsymbol{M}_{\mathbf{1}}\right|}\right)+\frac{6}{b}+\frac{1}{4 d}\right]
$$

and

$$
\operatorname{trace}(M(\mathrm{~T}))^{0}=\operatorname{trace} \mathbf{I}_{6}=6
$$

now using (12) and (13) in (9), with $\alpha_{1}+\alpha_{2}=1$, we obtain

$$
\alpha_{1}^{5}-11.6781 \alpha_{1}^{4}-40.7289 \alpha_{1}^{3}-46.8974 \alpha_{1}^{2}+16.84662 \alpha_{1}=0
$$

This gives $\alpha_{1}=0.283748894$ for $\alpha_{1} \in[0,1]$ and $\alpha_{2}=1-0.283748894=0.716251105$

Thus using (3) in (9) together with values of $\alpha_{1}$ and $\alpha_{2}$ from (14), the unique D - optimal moment matrix for $\{2,2\}$ WRSD is

$$
\left(\begin{array}{cccc}
1 & 0.41661_{2}^{\prime} & 0 & 0 \\
0.41661_{2} & 0.2141 \mathrm{I}_{2}+0.1432\left(e_{1} e_{2}^{\prime}+e_{2} e_{1}^{\prime}\right) & 0 & 0 \\
0 & 0 & 0.4166 \mathrm{I}_{2} & 0 \\
0 & 0 & 0 & 0.1432
\end{array}\right)
$$

\subsubsection{A-Optimal Wrsd}

A convex combination

$$
\eta(\alpha)=\sum_{j=1}^{m} \alpha_{j} \eta_{j}
$$

with

$$
\alpha=\left(\alpha_{1}, \alpha_{2}, \ldots \ldots \ldots, \alpha_{m}\right)^{\prime} \in \mathrm{T}
$$

is a weighted design with weight vector $\alpha_{i}$ where

$$
\sum_{i=1}^{m} \alpha_{i}=1
$$

From the Kiefer-Wolfowitz equivalence theorem in Pukelsheim (1993), if $\eta(\alpha)$ satisfies the side condition $C_{k}(M(\eta(\alpha))) \in P D(s)$ and $C_{i}$ written as $C_{i}=C_{k}\left(M\left(\eta_{i}\right)\right)$ Trace $C_{i} C_{k}(M(\eta(\alpha)))^{-2}=\operatorname{trace} C_{k}(M(\eta(\alpha)))^{-1} i=1, \ldots \ldots, m$

Therefore, a WRSD $\eta(\alpha)$ is A-optimal if and only if (19) is satisfied

From (6), let $(M(\mathrm{~T}))^{-1}=\left(\begin{array}{cl}V^{-1} & 0 \\ 0 & W^{-1}\end{array}\right)$ so that

$$
(M(\mathrm{~T}))^{-2}=\left(\begin{array}{cl}
V^{-2} & 0 \\
0 & W^{-2}
\end{array}\right)
$$

Where $\quad V=\left(\begin{array}{cc}1 & b 1_{2}^{\prime} \\ b 1_{2} & c \mathrm{I}_{2}+d\left(e_{1} e_{2}^{\prime}+e_{2} e_{1}^{\prime}\right)\end{array}\right)$ and $W=$ $\left(\begin{array}{cc}b \mathrm{I}_{2} & 0 \\ 0 & d\end{array}\right)$

This gives for $i=1, \ldots \ldots, m$, then, $\eta(\alpha)$ Solves the design problem i.e the $\theta_{\rho}$ optimal design problem if for $\rho=-1$

$$
V^{-1}=\left(\begin{array}{cc}
e & f 1_{2}^{\prime} \\
f 1_{2} & g \mathrm{I}_{2}+h\left(e_{1} e_{2}^{\prime}+e_{2} e_{1}^{\prime}\right)
\end{array}\right) \text { and } W^{-1}=\left(\begin{array}{cc}
\frac{1}{b} \mathrm{I}_{2} & 0 \\
0 & \frac{1}{d}
\end{array}\right)
$$

Similarly, $\left(V^{-1}\right)^{2}=\left(\begin{array}{cc}2 f^{2}+e^{2} & f(h+g+e) 1_{2}^{\prime} \\ f(h+g+e) 1_{2} & \left(h^{2}+g^{2}+f^{2}\right) \mathrm{I}_{2}+\left(2 g h+f^{2}\right)\left(e_{1} e_{2}^{\prime}+e_{2} e_{1}^{\prime}\right)\end{array}\right)$ and

$$
\left(W^{-1}\right)^{2}=\left(\begin{array}{cc}
\frac{1}{b^{2}} \mathrm{I}_{2} & 0 \\
0 & \frac{1}{d^{2}}
\end{array}\right)
$$

from (21), $M(\mathrm{~T})^{-2}$ then becomes 


$$
\left(\begin{array}{cccc}
p & q 1_{2}^{\prime} & 0 & 0 \\
q 1_{2} & r \mathrm{I}_{2}+s\left(e_{1} e_{2}^{\prime}+e_{2} e_{1}^{\prime}\right) & 0 & 0 \\
0 & 0 & \frac{1}{b^{2}} \mathrm{I}_{2} & 0 \\
0 & 0 & 0 & \frac{1}{d^{2}}
\end{array}\right)
$$

Where

$$
p=2 f^{2}+e^{2}, \quad q=f(h+g+e), \quad r=h^{2}+g^{2}+f^{2} \text { and } \quad s=2 g h+f^{2}
$$

In which;

$$
e=\frac{c^{2}-d^{2}}{|\boldsymbol{V}|}, \quad f=\frac{-b(c-d)}{|\boldsymbol{V}|}, \quad g=\frac{a c-b^{2}}{|\boldsymbol{V}|} \text { and } \quad h=\frac{b^{2}-a d}{|\boldsymbol{V}|}
$$

Thus $M\left(\tau_{1}\right)\left(M(\mathrm{~T})^{-2}\right)$ becomes

$$
\frac{1}{8}\left(\begin{array}{cccc}
8 p+6 q & (8 q+3 r+3 s) 1_{2}^{\prime} & 0 & 0 \\
\left(3 p+\frac{10}{4} q\right) 1_{2} & \left(3 q+\frac{9}{4} r+\frac{1}{4} s\right) \mathrm{I}_{2}+\left(3 q+\frac{9}{4} s+\frac{1}{4} r\right)\left(e_{1} e_{2}^{\prime}+e_{2} e_{1}^{\prime}\right) & 0 & 0 \\
0 & 0 & \frac{3}{b^{2}} \mathrm{I}_{2} & 0 \\
0 & 0 & 0 & \frac{1}{4 d^{2}}
\end{array}\right)
$$

and

$$
\operatorname{trace}\left(M\left(\tau_{1}\right)\left(M(\mathrm{~T})^{-2}\right)\right)=\frac{1}{8}\left(8 p+12 q+\frac{9}{2} r+\frac{1}{2} s+\frac{6}{b^{2}}+\frac{1}{4 d^{2}}\right)
$$

$$
\begin{aligned}
p & =\frac{\alpha_{1}^{2}}{(64|V|)^{2}}\left[\left(6 \alpha_{1}+6.9282 \alpha_{2}\right)^{2}+\left(5 \alpha_{1}+6 \alpha_{2}\right)^{2}\right] \\
q & =\frac{\alpha_{1}^{2}}{(64|V|)^{2}}\left[\left(6 \alpha_{1}+6.9282 \alpha_{2}\right)^{2}\left[\left(7 \alpha_{1}+6.7846 \alpha_{2}\right)+\left(9 \alpha_{1}+9.2154 \alpha_{2}\right)+\left(5 \alpha_{1}+6 \alpha_{2}\right)^{2}\right]\right] \\
\text { with } r & =\frac{\alpha_{1}^{2}}{\left(\left.64|V|\right|^{2}\right.}\left[\left(7 \alpha_{1}+6.7846 \alpha_{2}\right)^{2}+\left(9 \alpha_{1}+9.2154 \alpha_{2}\right)^{2}+\left(6 \alpha_{1}+6.9282 \alpha_{2}\right)^{2}\right] \\
s & =\frac{\alpha_{1}^{2}}{(64|V|)^{2}}\left[\left(9 \alpha_{1}+9.2154 \alpha_{2}\right)\left(7 \alpha_{1}+6.7846 \alpha_{2}\right)+\left(6 \alpha_{1}+6.9282 \alpha_{2}\right)^{2}\right] \\
|V| & =\frac{1}{4} \alpha_{1}\left[\frac{2 \alpha_{1}^{2}+2.4308 \alpha_{1} \alpha_{2}}{64}\right]
\end{aligned}
$$

Now from [20],

$$
\operatorname{trace}\left(M(\mathrm{~T})^{-1}\right)=e+2 g+\frac{2}{b}+\frac{1}{d}
$$

Using (25) and (26) in (19) results in

$$
\begin{gathered}
1+11.0408 \alpha_{1}+18.1390 \alpha_{1}^{2}-55.7918 \alpha_{1}^{3}-214.6311 \alpha_{1}^{4}-1189.2271 \alpha_{1}^{5}-2567.6833 \alpha_{1}^{6}+2528.7509 \alpha_{1}^{7}- \\
1273.3248 \alpha_{1}^{8}-254.5424 \alpha_{1}^{9}=0
\end{gathered}
$$

Which when solved within $[0,1]$ we get $\alpha_{1}=0.279637843$ and $\alpha_{2}=1-\alpha_{1}=0.720362156$

Hence the unique A-optimal design is

$$
\eta\left(\alpha^{A}\right)=0.279637843 \tau_{1}+0.720362156 \tau_{2}
$$

using (4) and (5) together with $\alpha_{1}$ and $\alpha_{2}$ values from (27) in (9), we have

$$
\left(\begin{array}{cccc}
1 & 0.41681_{2}^{\prime} & 0 & 0 \\
0.41681_{2} & 0.2137 \mathrm{I}_{2}+0.1438\left(e_{1} e_{2}^{\prime}+e_{2} e_{1}^{\prime}\right) & 0 & 0 \\
0 & 0 & 0.4168 \mathrm{I}_{2} & 0 \\
0 & 0 & 0 & 0.1438
\end{array}\right)
$$

the required A-optimal design. 


\subsection{Construction of $\{3,2\}$ Wrsd}

Similar to 3.1 above, the elementary RSD $\tau_{1}$ and $\tau_{2}$ are used to generate the WRSD T such that: For weights $\alpha_{1}, \alpha_{2} \geq 0$ with $\alpha_{1}+\alpha_{2}=1$, the design $\mathrm{T}=\alpha_{1} \tau_{1}+\alpha_{2} \tau_{2}$

Has the moment matrix (2) i.e. $M(\mathrm{~T})=\alpha_{1} M\left(\tau_{1}\right)+\alpha_{2} M\left(\tau_{2}\right)$

Where

$$
\begin{gathered}
t_{0 u} \\
\tau_{1}
\end{gathered}=\left[\begin{array}{rrrr}
1 & t_{2 u} & t_{3 u} \\
1 & -1 & 0 & 0 \\
1 & 0 & 1 & 0 \\
1 & 0 & -1 & 0 \\
1 & 0 & 0 & 1 \\
1 & 0 & 0 & -1 \\
1 & \frac{1}{2} & \frac{1}{2} & 0 \\
1 & -\frac{1}{2} & \frac{1}{2} & 0 \\
1 & \frac{1}{2} & -\frac{1}{2} & 0 \\
1 & -\frac{1}{2} & -\frac{1}{2} & 0 \\
1 & \frac{1}{2} & 0 & \frac{1}{2} \\
1 & -\frac{1}{2} & 0 & \frac{1}{2} \\
1 & \frac{1}{2} & 0 & -\frac{1}{2} \\
1 & -1 & 0 & -1
\end{array}\right] \text { and } \tau_{2}=\left[\begin{array}{rrrr}
t_{0 u} & t_{1 u} & t_{2 u} & t_{3 u} \\
1 & -a & -a & -a \\
1 & -a & a & -a \\
1 & -a & a & a \\
1 & a & -a & -a \\
1 & a & -a & a \\
1 & a & a & -a \\
1 & a & a & a
\end{array}\right]
$$

From (30) we have:

$$
M\left(\tau_{1}\right)=\frac{1}{18}\left(\begin{array}{cccc}
18 & 41_{3}^{\prime} & 0 & 0 \\
41_{3} & \frac{9}{4} \mathrm{I}_{3}+\frac{1}{4} \mathrm{~J}_{3} & 0 & 0 \\
0 & 0 & 4 \mathrm{I}_{3} & 0 \\
0 & 0 & 0 & \frac{1}{4} \mathrm{I}_{3}
\end{array}\right)
$$

and

$$
M\left(\tau_{2}\right)=\frac{1}{8}\left(\begin{array}{cccc}
8 & \left(8 a^{2}\right) 1_{3}^{\prime} & 0 & 0 \\
\left(8 a^{2}\right) 1_{3} & \left(8 a^{4}\right) \mathrm{J}_{3} & 0 & 0 \\
0 & 0 & \left(8 a^{2}\right) \mathrm{I}_{3} & 0 \\
0 & 0 & 0 & \left(8 a^{4}\right) \mathrm{I}_{3}
\end{array}\right)
$$

So that (2) becomes

$$
\left(\begin{array}{cccc}
1 & \left(\frac{2 \alpha_{1}}{9}+a^{2} \alpha_{2}\right) 1_{3}^{\prime} & 0 & 0 \\
\left(\frac{2 \alpha_{1}}{9}+a^{2} \alpha_{2}\right) 1_{3} & \frac{1}{8} \mathrm{I}_{3}+\left(\frac{\alpha_{1}}{72}+a^{4} \alpha_{2}\right) \mathrm{I}_{3} & 0 & 0 \\
0 & 0 & \left(\frac{2 \alpha_{1}}{9}+a^{2} \alpha_{2}\right) \mathrm{I}_{3} & 0 \\
0 & 0 & 0 & \left(\frac{\alpha_{1}}{72}+a^{4} \alpha_{2}\right) \mathrm{I}_{3}
\end{array}\right)
$$

Where $a=\left[\frac{7}{64}\right]^{\frac{1}{4}}$ 


\section{4. $\{3,2\}$ Optimal Designs}

Similarly, the general equivalence theorem is used to compute the values of the masses assigned to the design points of the RSD to obtain D-optimal and A-optimal WRSDs

\subsubsection{D-Optimal Wrsd}

D - optimal WRSD design is:

$$
\eta\left(\alpha^{(D)}\right)=\alpha_{1} \tau_{1}+\alpha_{2} \tau_{2}
$$

such that:

$$
M(\mathrm{~T})=\alpha_{1} M\left(\tau_{1}\right)+\alpha_{2} M\left(\tau_{2}\right)
$$

Using the GET to obtain values of $\alpha_{1}$ and $\alpha_{2}$ which would give a D-optimal design. i.e solving $\alpha_{1}$ and $\alpha_{2}$ to satisfy

$$
\operatorname{trace}\left(M\left(\tau_{i}\right)(M(\mathrm{~T}))^{-1}\right)=\operatorname{trace}(M(\mathrm{~T}))^{0} \text { for } i=1,2
$$

$M\left(\tau_{1}\right)(M(\mathrm{~T}))^{-1}$ is as in APENDIX I

$$
\begin{gathered}
\therefore \operatorname{trace}\left(M\left(\tau_{1}\right)(M(\mathrm{~T}))^{-1}\right) \\
=\frac{1}{18}\left[\frac{18(c-d)^{2}(c+2 d)-12 b(c-d)^{2}}{|V|}+\frac{3}{|V|}\left[\frac{5(c-d)\left[a(c+d)-2 b^{2}\right]}{2}+\frac{\left(b^{2}-a d\right)(c-d)}{2}-4 b(c-d)^{2}\right]+\frac{12}{b}+\frac{3}{4 d}\right]
\end{gathered}
$$

and

$$
\operatorname{trace}(M(\mathrm{~T}))^{0}=\operatorname{trace} \mathrm{I}_{10}=10
$$

now using (36) and (37) in (34) with $\alpha_{1}+\alpha_{2}=1$, we obtain

$$
1-3.564144 \alpha_{1}+5.924622 \alpha_{1}^{2}-5.2839 \alpha_{1}^{3}+3.4871 \alpha_{1}^{4}-4.36076 \alpha_{1}^{5}+0.5915 \alpha_{1}^{6}=0
$$

This gives $\alpha_{1}=0.596127182$ for $\alpha_{1} \in[0,1]$, and $\alpha_{2}=1-0.596127182=0.403872817$

Therefore using (30) with $\alpha_{1}$ and $\alpha_{2}$ values from (38) in (34), the unique $\mathrm{D}$ - optimal moment matrix for $\{3,2\}$ WRSD is

$$
\left(\begin{array}{cccc}
1 & 0.26601_{3}^{\prime} & 0 & 0 \\
0.26601_{3} & 0.0745 \mathrm{I}_{3}+0.0525 \mathrm{~J}_{3} & 0 & 0 \\
0 & 0 & 0.2660 \mathrm{I}_{3} & 0 \\
0 & 0 & 0 & 0.0525 \mathrm{I}_{3}
\end{array}\right)
$$

\subsubsection{A - Optimal Wrsd}

Similarly, a WRSD $\eta(\alpha)$ is A-optimal if and only if (18) is satisfied

From (32), let $M(\mathrm{~T})=\left(\begin{array}{cc}V & 0 \\ 0 & W\end{array}\right)$ where;

$$
V=\left(\begin{array}{cc}
1 & \left(\frac{2 \alpha_{1}}{9}+a^{2} \alpha_{2}\right) 1_{3}^{\prime} \\
\left(\frac{2 \alpha_{1}}{9}+a^{2} \alpha_{2}\right) 1_{3} & \frac{1}{8} \mathrm{I}_{3}+\left(\frac{\alpha_{1}}{72}+a^{4} \alpha_{2}\right) \mathrm{J}_{3}
\end{array}\right) \text { and } W=\left(\begin{array}{cl}
\left(\frac{2 \alpha_{1}}{9}+a^{2} \alpha_{2}\right) \mathrm{I}_{3} & 0 \\
0 & \left(\frac{\alpha_{1}}{72}+a^{4} \alpha_{2}\right) \mathrm{I}_{3}
\end{array}\right)
$$

Such that

$$
(M(\mathrm{~T}))^{-1}=\left(\begin{array}{cl}
V^{-1} & 0 \\
0 & W^{-1}
\end{array}\right) \text { then }(M(\mathrm{~T}))^{-2}=\left(\begin{array}{cl}
V^{-2} & 0 \\
0 & W^{-2}
\end{array}\right)
$$

With $V^{-1}=\left(\begin{array}{cc}e & f 1_{3}^{\prime} \\ f 1_{3} & (h-g) \mathrm{I}_{3}+g \mathrm{~J}_{3}\end{array}\right)$ and $W^{-1}=\left(\begin{array}{cc}\frac{1}{b} \mathrm{I}_{3} & 0 \\ 0 & \frac{1}{d} \mathrm{I}_{3}\end{array}\right)$

Then 


$$
\begin{aligned}
\left(V^{-1}\right)^{2} & =\left(\begin{array}{cc}
3 f^{2}+e^{2} & (f(2 h+g+e)) 1_{3}^{\prime} \\
(f(2 h+g+e)) 1_{3} & (h-g)^{2} \mathrm{I}_{3}+\left(h^{2}+2 g h+f^{2}\right) \mathrm{I}_{3}
\end{array}\right) \text { and } \\
\left(W^{-1}\right)^{2} & =\left(\begin{array}{cc}
\frac{1}{b^{2}} \mathrm{I}_{3} & 0 \\
0 & \frac{1}{d^{2}} \mathrm{I}_{3}
\end{array}\right)
\end{aligned}
$$

Thus trace $\left(M\left(\tau_{1}\right)(M(\mathrm{~T}))^{-2}\right)$ is

$$
\frac{1}{18}\left[\frac{1}{|V|^{2}}\left\{24 e f+24 f g+48 f h+18 e^{2}+63 f^{2}+3 g h+\frac{15}{2} g^{2}+\frac{33}{2 h^{2}}\right\}+\frac{12}{b^{2}}+\frac{3}{4 d^{2}}\right]
$$

Where

$$
\begin{array}{lll}
e=(c-d)^{2}(c+2 d), & f=-b(c-d)^{2}, & g=(c-d)\left[(c+d)-2 b^{2}\right] \\
h=(c-d)\left(b^{2}-d\right) \text { and } & |V|=(c-d)^{2}\left[(c+d)-2 b^{2}\right] &
\end{array}
$$

With

$$
\begin{aligned}
& b=\frac{2}{9} \alpha_{1}+\left(\frac{7}{64}\right)^{\frac{1}{2}} \alpha_{2}, \quad c=\frac{5}{36} \alpha_{1}+\frac{7}{64} \alpha_{2} \text { and } \quad d=\frac{\alpha_{1}}{72}+\frac{7}{64} \alpha_{2} \\
& \text { Now from (A2), } \operatorname{tr} \text { a c } e\left(M(\mathrm{~T})^{-1}\right)=\frac{e}{|V|}+\frac{3 g}{|V|}+\frac{3}{b}+\frac{3}{d}
\end{aligned}
$$

Using (42) and (43) in (19) results in

$$
\begin{gathered}
\alpha_{1}^{11}-21.8288 \alpha_{1}^{10}+44.0225 \alpha_{1}^{9}-494.7780 \alpha_{1}^{8}+283.3541 \alpha_{1}^{7}-236.8855 \alpha_{1}^{6}+233.7964 \alpha_{1}^{5}-55.2344 \alpha_{1}^{4}- \\
166.1101 \alpha_{1}^{3}+35.0103 \alpha_{1}^{2}+0.4652 \alpha_{1}=0
\end{gathered}
$$

Which when solved within $[0,1]$ we get $\alpha_{1}=0.219614833$ and

$$
\alpha_{2}=1-\alpha_{1}=0.780385166
$$

Hence the unique A-optimal design is

$$
\eta\left(\alpha^{A}\right)=0.219614833 \eta_{1}+0.780385166 \eta_{2}
$$

Using values of $\alpha_{1}$ and $\alpha_{2}$ from (44) in (30) we have;

$$
\left(\begin{array}{cccc}
1 & 0.30691_{3}^{\prime} & 0 & 0 \\
0.30691_{3} & 0.0275 \mathrm{I}_{3}+0.0884 \mathrm{~J}_{3} & 0 & 0 \\
0 & 0 & 0.3069 \mathrm{I}_{3} & 0 \\
0 & 0 & 0 & 0.0886 \mathrm{I}_{3}
\end{array}\right)
$$

the required A-optimal design.

\section{Efficiencies of the Designs}

The performance of the RSD and WRSD was measured using the $\mathrm{D}$ - and $\mathrm{A}$ - criterion.

Table 2. Optimal values.

\begin{tabular}{lllll}
\hline & \multicolumn{2}{l}{$\begin{array}{l}\text { Rotatable Simplex Design } \\
\text { (RSD) }\end{array}$} & \multicolumn{2}{l}{$\begin{array}{l}\text { Weighted Rotatable } \\
\text { Simplex Design (WRSD) }\end{array}$} \\
\hline Factors $(\boldsymbol{k})$ & $\boldsymbol{D}-$ & $\boldsymbol{A}-$ & $\boldsymbol{D}-$ & $\boldsymbol{A}-$ \\
\hline 2 & 0.1908 & 2.5295 & 0.1618 & 0.0372 \\
3 & 0.1075 & 4.3744 & 0.0258 & 0.0409 \\
\hline
\end{tabular}

\subsection{D - Efficiency}

The performance of the WRSD in comparison to the RSD is measured by the D-efficiency which is defined by
$(\tau)=\left\{\frac{|M(\tau)|}{\left|M\left(\tau^{0}\right)\right|}\right\}^{\frac{1}{s}}$. Using the D- optimal $\emptyset_{0}\left(C_{k}(M)\right)$ values for the two designs from Table 1, the D - Efficiency values are:

Table 3. D-Efficiency values.

\begin{tabular}{llll}
\hline $\begin{array}{l}\text { Design } \\
\text { Factors }\end{array}$ & $\begin{array}{l}\text { Rotatable } \\
\text { Simplex Design } \\
\text { (RSD) }\end{array}$ & $\begin{array}{l}\text { Weighted } \\
\text { Rotatable Simplex } \\
\text { Design (WRSD) }\end{array}$ & $\begin{array}{l}\text { D - efficiency } \\
\boldsymbol{D}_{\text {eff }}\left(\boldsymbol{\eta}^{*}\right)\end{array}$ \\
\hline 2 & 0.1908 & 0.1618 & 0.8480 \\
3 & 0.1075 & 0.0258 & 0.24 \\
\hline
\end{tabular}

From the efficiencies, it is noted that the WRSD is $15.2 \%$ more D - efficient for two factors and $76 \%$ more D - efficient for three factors. 


\subsection{A - Efficiency}

Similarly the A - optimal $\emptyset_{-1}\left(C_{k}(M)\right)$ values for the two designs from Table 1 are used to obtain the A - Efficiency values as $\frac{\emptyset_{-1}\left(C_{1}\right)}{\emptyset_{-1}\left(C_{2}\right)}=\frac{\left(\frac{1}{s} \text { trace }_{1}^{-1}\right)^{-1}}{\left(\frac{1}{s} \text { trace }_{2}^{-1}\right)^{-1}}$ :

Table 4. A-Efficiency values.

\begin{tabular}{llll}
\hline $\begin{array}{l}\text { Design } \\
\text { Factors }\end{array}$ & $\begin{array}{l}\text { Rotatable } \\
\text { Simplex Design } \\
\text { (RSD) }\end{array}$ & $\begin{array}{l}\text { Weighted Rotatable } \\
\text { Simplex Design } \\
\text { (WRSD) }\end{array}$ & $\begin{array}{l}\text { A - efficiency } \\
\boldsymbol{A}_{\text {eff }}\left(\boldsymbol{\eta}^{*}\right)\end{array}$ \\
\hline 2 & 2.5295 & 0.0372 & 0.0147 \\
3 & 4.3744 & 0.0409 & 0.0093 \\
\hline
\end{tabular}

From the efficiencies, it is also noted that the WRSD is 98.53\% more A - efficient for two factors and $99.07 \%$ more
A - efficient for three factors.

\section{Conclusion}

In this study, we have presented a method for constructing a WRSD. The constructed design has achieved estimation efficiency as shown by the results in relation to their moment matrices. These designs have also proved to be D- and Aoptimal.

\section{Appendix}

I. Matrices for $\{3,2\}$ RSD A - Optimality

$$
M\left(\tau_{1}\right)(M(\mathrm{~T}))^{-1} \text { for }\{3,2\} \mathrm{RSD}
$$

$$
M\left(\tau_{1}\right)(M(\mathrm{~T}))^{-1}=\frac{1}{18}\left[\begin{array}{cccc}
18 & 41_{2}^{\prime} & 0 & 0 \\
41_{2} & \frac{9}{4} \mathrm{I}_{3}+\frac{1}{4} \mathrm{~J}_{3} & 0 & 0 \\
0 & 0 & 4 \mathrm{I}_{3} & 0 \\
0 & 0 & 0 & \frac{1}{4} \mathrm{I}_{3}
\end{array}\right]\left[\begin{array}{cc}
V^{-1} & 0 \\
0 & W^{-1}
\end{array}\right]
$$

Where

With

$$
V^{-1}=\left(\begin{array}{cc}
(c-d)^{2}(c+2 d) & \left(-b(c-d)^{2}\right) 1_{3}^{\prime} \\
\left(-b(c-d)^{2}\right) 1_{3} & |V| \mathrm{I}_{3}+(c-d)\left(b^{2}-a d\right) \mathrm{J}_{3}
\end{array}\right)
$$

$$
|V|=(c-d)^{2}\left[a(c+2 d)-3 b^{2}\right] \text { and } \quad W^{-1}=\left(\begin{array}{cc}
\frac{1}{b} \mathrm{I}_{3} & 0 \\
0 & \frac{1}{d} \mathrm{I}_{3}
\end{array}\right)
$$

\section{References}

[1] Box, G. E. P., \& Draper, N. R. (1959). A basis for the selection of a response surface design. Journal of American Statistical Association, 54, 622-654.

[2] Das, M. N., \& Narasimham, V. L. (1962). Construction of rotatable designs through balanced incomplete block designs. Annals of Mathematical Statistics, 33(4), 1421-1439.

[3] Das, R. N. (1997). Robust second order rotatable designs: Part I RSORD. Calcutta Statistical Association Bulletin, 47, 199-214.

[4] Das, R. N. (1999). Robust Second Order Rotatable Designs: Part II RSORD. Calcutta Statistical Association Bulletin, 49, 65-76.

[5] Otieno-Roche E., Koske J. \& Mutiso J. (2017). Construction of Second Order Rotatable Simplex Designs. Manuscript submitted for publication.

[6] Panda, R. N., \& Das, R. N. (1994). First order rotatable designs with correlated errors. Calcutta Statistical Association Bulletin, 44, 83-101.

[7] Rajyalakshmi, K., \& Victorbabu B. R. (2014). Construction of second order rotatable designs under tri-diagonal correlation structure of errors using central composite designs. Journal of Statistics: Advances in Theory and Applications, 11(2), 71-90.

[8] Rajyalakshmi, K., \& Victorbabu, B. R. (2011). Robust Second Order Rotatable Central Composite Designs. JP Journal of Fundamental and Applied Statistics, 1(2), 85-102.

[9] Tyagi, B. N. (1964). Construction of second order and third order rotatable designs through pairwise balanced designs and doubly balanced designs. Calcutta Statistical Association Bulletin, 13, 150-162.

[10] Victorbabu, B. R., \& Rajyalakshmi, K. (2012). A new method of construction of robust second order rotatable designs using balanced incomplete block designs. Open Journal of Statistics, 2(2), 88-96.

[11] Victorbabu, B. R., \& Rajyalakshmi, K. (2012). Robust second order slope rotatable designs using balanced incomplete block designs. Open Journal of Statistics, 2(2), 65-77. 